\title{
MRSA and Inducible Clindamycin resistance in Staphylococcus aureus from Various Samples in A Tertiary Care Hospital
}

\author{
R. Krishnan, M. S. Harbade (Duthade)", J. A. Iravane (Bajaj) and A. A. Gaikwad
}

Department of Microbiology, Government Medical College, Aurangabad, Maharashtra, India

*Corresponding author

\section{A B S T R A C T}

Keywords

Clindamycin resistance, Patient, Staphylococcus,

Methicillin

resistance,

Outbreaks

Article Info

Accepted:

04 November 2019

Available Online:

10 December 2019
The study was done to note the presence of MRSA and Inducible resistance to Clindamycin in various samples of the Staphylococcus aureus from the Hospital. A total of 1607 of Staphylococci were isolated from various clinical samples like Pus, Wound swab, Body fluids, Urine, Vaginal swab, Throat swab and other samples coming from hospital. Among the 1607 clinical isolates of Staphylococcus aureus MRSA (Methicillin resistance Staphylococcus aureus) were seen in 695 (43.2\%). Among this 188 (97MRSA, 91MSSA) isolates showed Inducible Clindamycin resistance that is resistance to Erthromycin and sensitive to Clindamycin sensitive showing D test positive. 524 (294MRSA; 230MSSA) showed Constitutive MLsB resistance that is resistance to both Erythromycin and Clindamycin. The resistance of inducible Clindamycin and Constitutive MLsB was more in MRSA than in Methicillin Sensitive Staphylococcus aureus. In addition to MRSA and inducible Clindamycin resistance other antibiotics were also noted.

\section{Introduction}

Among the genus Staphylococcus the most pathogenic is Staphylococcus aureus. This organism is responsible for causing suppurative types of infections. They have ability to spread and cause outbreaks in hospitals. Most Staphylococcus aureus infections resolve spontaneously or in response to antibiotic treatment, but in recent years there have been increasing in developing resistance to multiple antibiotics (Srinivasan et al., 2002).

Among the commonest resistance is Methicillin resistance Staphylococcus aureus (MRSA). MRSA is responsible for causing outbreaks in hospital. MRSA resistance was first noted in 1961. Methicillin resistance is usually confirmed by altered penicillin 
binding protein that causes resistance to all Blactam antimicrobial agents (Fishovitz et al., 2014). Although a predominantly a hospital pathogen, MRSA is becoming more common in community infections. Hospital personnel harboring MRSA act as chief source of nosocomial infections. The other predisposing factors that increase the chance of emergence and spread of MRSA are prolonged and repeated hospitalization, indiscriminate use of antibiotics, and lack of awareness, intravenous drug abuse, and presence of indwelling medical devices (Weisblum et al., 1985).

The resistance in Staphylococcus is also seen with Erythromycin (ERY) (a Macrolide) and Clindamycin (CLI) (a Lincosamide). These two drugs represent two distinct classes of antimicrobial agents that inhibit protein synthesis by binding to the $50 \mathrm{~S}$ ribosomal subunits of bacterial cells. Resistance to both of these antimicrobial agents can occur through methylation of their ribosomal target site. Such resistance is typically mediated by erm genes. Resistance to macrolides also can occur by efflux, typically mediated by the $m s r A$ gene. An in vitro induction test can distinguish Staphylococci that have inducible erm-mediated resistance from those with $m s r A$ mediated resistance. Methicillin-resistant Staphylococcus aureus (MRSA)are increasingly being reported as multidrug resistant with high resistance to Macrolides (Erythromycin, Clarithromycin) and Lincosamides (Clindamycin, Lincomycin) leaving very few therapeutic options.

Main aim of the study was to see the presence of MRSA and inducible Clindamycin resistance in Staphylococcus aureus.

\section{Materials and Methods}

The study was conducted in the Bacteriology section of Microbiology Department of one year from June 2017 to June 2018 in
Government Medical College Aurangabad. During this period 1607 Staphylococci aureus were isolated from various clinical samples like blood culture, urine, pus, vaginal swab, throat swabs, body fluids and others (sputum, aspirates, respiratory, central line/neck line/umbilical catheter tips) were included in the study.

Isolation of Staphylococcus aureus were done by conventional bacteriological methods such as colony morphology, Gram staining, Catalase, Coagulase test. All samples were processed as per standard procedures.

Antibiotic sensitivity testing of Staphylococcus aureus was done using Modified Kirby Bauer disc diffusion method (CLSI guidelines Jan 2017).

The isolates were tested for Cefoxitin $(30 \mu \mathrm{g})$, Amoxillin, Azithromycin (15 $\mu \mathrm{g})$, Erythromycin (15 $\mu \mathrm{g}), \quad$ Cotrimaxazole $(1.25 / 23.75 \mu \mathrm{g})$, Ciprofloxacillin $(5 \mu \mathrm{g})$, Clindamycin, Vancomycin and Linezolid (30 $\mu \mathrm{g})$.

The Staphylococcus aureus isolates were assessed for Methicillin and Clindamycin resistance. Methicillin resistance was determined by using Cefoxitin antibiotic $(30 \mu \mathrm{g})$ disc. In our study MRSA were 695 $(43.2 \%)$ and MSSA were $912(56.7 \%)$ as shown in Table 1.

Maximum number of MRSA were found in Pus 340 (48.9\%), Blood 194 (27.9\%), body fluids $50(7.1 \%)$, Urine $20(2.8 \%)$, throat swabs $20(2.8 \%)$, Vaginal $9(1.2 \%)$ and others $62(8.9 \%)$ as shown in Table 2.

Detection of Inducible Clindamycin test is done on same plate by placing Clindamycin $(2 \mu \mathrm{g})$ discs is placed $15 \mathrm{~mm}$ from an Erythromycin $(15 \mu \mathrm{g})$ adjacent to each other on Mueller-Hinton agar. Flattening of the 
Clindamycin zone suggests inducible Clindamycin resistance (Watanakunakorn et al., 1976). According to studies, D test should be used as a mandatory method in routine disc diffusion testing to detect inducible Clindamycin resistance in Staphylococci for the optimum treatment of patients.

Out of 1607 staphylococcus aureus (695 MRSA; 912 MSSA) were isolates. Out of this 355 (64 MRSA; 291MSSA) were susceptible to Erthryomycin and Clindamycin. 524 (294MRSA; 230MSSA) showed Constitutive MLsB resistance that is resistance to both Erythromycin and Clindamycin.

188 (97 MRSA, 91 MSSA) isolates showed Inducible Clindamycin resistance that is resistance to Erthromycin and sensitive to Clindamycin sensitive showing D test positive as shown in Table 3. The resistance of inducible Clindamycin was more in MRSA that is $97(13.9 \%)$ than in MSSA.

And those showing MS Phenotype 540(240 MRSA; 300 MSSA) showed Erythromycin resistance and Clindamycin sensitive and D test negative.

In addition to MRSA and inducible Clindamycin resistance other antibiotics also shows resistance. These drugs were Amoxclav (79\%), Co-trimoxazole (56\%) and Gentamycin (40.2\%). However, all (100\%) isolates were susceptible to Vancomycin and Linezolid.

\section{Results and Discussion}

Staphylococcus aureus is a common human pathogen that causes a wide variety of infections. MRSA is a major Nosocomial pathogen causing significant morbidity and mortality (Sachdev et al., 2003). In our study
MRSA was found to be $43.4 \%$ of the total Staphylococcus aureus isolates, similar type of study was done by Mittal S et al., had reported the same percentage of MRSA (Mittal et al., 2019).

In our study among the various samples maximum number of MRSA were found in Pus 340 (48.9\%) samples. Similar observation was made by Rupali Mantri et al., (2014) in Akola and reported high isolation rate of upto $67.85 \%$ of MRSA strains from pus specimens

188 (97MRSA, 91MSSA) isolates showed Inducible Clindamycin resistance that is resistance to Erthromycin and sensitive to Clindamycin showing D test positive. 524 (294MRSA; 230MSSA) showed Constitutive MLsB resistance that is resistance to both Erythromycin and Clindamycin. The resistance of inducible Clindamycin 97 (13.9\%) and 294 (56.1\%) Constitutive MLsB resistance was more in MRSA than in MSSA. Similar observation was noted in Javeria Firdous and Patil (2019).

The determination of antimicrobial susceptibility of a clinical isolate is often crucial for optimal antimicrobial therapy. This is particularly important considering the increase of resistance and the emergence of multidrug resistant organisms Fiebelkorn KR et al., (2003)

The increasing incidence of MRSA brought the need for other effective antibiotics. Clindamycin is a drug which fits that role. It can be given orally. It is safe as well as effective. All these lead to the increased usage of Clindamycin and thereby development of resistance to it. This makes the detection of Clindamycin resistance important and useful (Fig. 1 and 2). 
Table.1 Distribution of MRSA in Staphylococcus aureus

\begin{tabular}{|c|c|c|}
\hline Staphylococcus isolates & Frequency & Percentage \\
\hline MRSA & 695 & $43.2 \%$ \\
\hline MSSA & 912 & $56.7 \%$ \\
\hline Total & 1607 & \\
\hline
\end{tabular}

Table.2 Distribution of Staphylococcus and MRSA in various samples

\begin{tabular}{|c|c|c|c|c|}
\hline Sr.no & Samples & $\begin{array}{c}\text { Total } \\
\text { samples }\end{array}$ & $\begin{array}{c}\text { Staphylococcus } \\
\text { aureus }\end{array}$ & MRSA \\
\hline $\mathbf{1}$ & Pus & 3373 & 600 & 340 \\
\hline $\mathbf{2}$ & Blood & 2872 & 543 & 194 \\
\hline $\mathbf{3}$ & Body fluids & 100 & 60 & 50 \\
\hline $\mathbf{4}$ & Urine & 1057 & 27 & 20 \\
\hline $\mathbf{5}$ & Throat swabs & 50 & 25 & 20 \\
\hline $\mathbf{6}$ & Vaginal swab & 70 & 20 & 9 \\
\hline $\mathbf{7}$ & Others. & 981 & 332 & 62 \\
\hline
\end{tabular}

Fig.1 MRSA with positive D-test

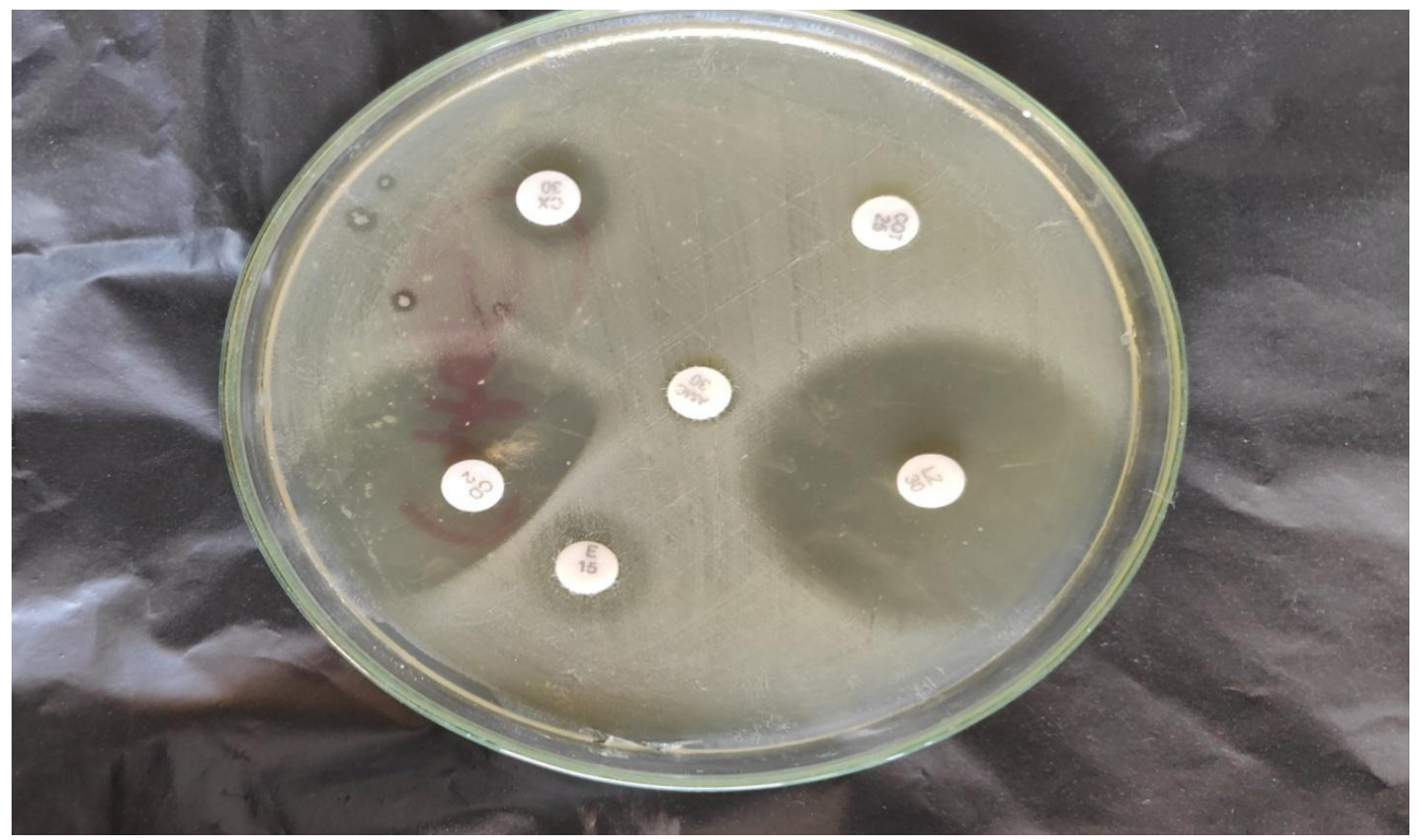


Fig.2 MRSA with negative D-test



Table.3 Erythromycin and clindamycin susceptibility pattern in Staphylococcus aureus isolates

\begin{tabular}{|c|c|c|c|c|}
\hline Sr.no & Susceptibility pattern & MRSA & MSSA & Total \\
\hline 1 & $\mathrm{CD}-\mathrm{S}$ & $64(9.2 \%)$ & $291(31.9 \%)$ & 355 \\
\hline 2 & $\begin{array}{cc}\text { E-R } & \text { CD-R } \\
\text { (Constitutive } & \text { MLSB) }\end{array}$ & $294(42.3 \%)$ & $230(25.2 \%)$ & 524 \\
\hline 3 & $\begin{array}{l}\text { E-R CD-S } \\
\text { D test positive } \\
\text { (Inducible } \\
\text { MLSB) }\end{array}$ & $97(13.9 \%)$ & $91(9.9 \%)$ & 188 \\
\hline 4 & $\begin{array}{l}\text { E-R CD-S } \\
\text { D test negative } \\
\text { ( MS Phenotype) }\end{array}$ & $240(34.5 \%)$ & $300(32.8 \%)$ & 540 \\
\hline 5 & Total & 695 & 912 & \\
\hline
\end{tabular}

Clindamycin is frequently used to treat skin and bone infections because of its tolerability, cost, oral form and excellent tissue penetration, and the fact that it accumulates in abscesses. Clindamycin is also used as an alternative for patients who are allergic to penicillin. MRSA isolates of our study were multidrug resistant. In addition to MRSA and Erthromycin resistance high antibiotic resistance to Amoxclav (79\%), Cotrimoxazole
$(56 \%)$ and Gentamycin (40.2\%) were noted. Vancomycin and Linozolid were found to be sensitive in MRSA and MSSA cases.

The degree of resistance or sensitivity of MRSA towards commonly used antimicrobials differs from one place to other. Therefore it is necessary to see the antibiotic sensitivity pattern in MRSA cases. In present study reliable method for detection of MRSA 
and inducible resistance to Clindamycin in isolates of Staphylococcus aureus were used. On this basis reporting of resistance to Cefoxitin and Inducible Clindamycin can be done on routine basis. We conclude that whenever Clindamycin is intended for Staphylococcus. aureus infection, the microbiology lab should tests the isolated organism for iMLSB by D test. D test is simple, inexpensive and easy to perform test. Clindamycin is drug of choice in case of D test negative isolates while it is not suitable for $\mathrm{D}$ test positive isolates.

\section{Acknowledgements}

All staff members of the Bacteriology Section of the Department of Microbiology, Govt. Medical. College, Aurangabad, Maharashtra.

\section{References}

Fiebelkorn KR, Crawford SA, McElmeel ML, Jorgensen JH. Practical disc diffusion method for detection of inducible Clindamycin resistance in Staphylococcus aureus and Coagulase negative Staphylococci. J Clin Microbiol 2003; 41: 4740-4.

Fishovitz J, Hermoso JA, Chang M, Mobashery S. Penicillin binding protein 2a of Methicillin resistant Staphylococcus aureus. IUBMB life. 2014; 66: 572-7.

Javeria Firdous, Basawaraj S. Patil. Study of Inducible Clindamycin Resistance in Staphylococcus aureus in a Tertiary care Hospital. International journal of current Microbiology and Applied Sciences. 2019. 8, No 03: 1471-1478.
Mittal S, Sayal P, Yadav P, Kumar A, Rajian M. Mupriocin Resistance among Methicillin resistant Staphylococcus isolates in tertiary health care centre. Infect Disord Drug Target.2019, 19(2): 128-132.

Clinical and Laboratory Standards Institute. Performance Standards for Antimicrobial Susceptibility Testing; Twenty-seventh Edition. CLSI document M100. Clinical and Laboratory Standards Institute, 940 West Valley Road, Suite 2500, Wayne, PA 19087 USA, Jan 2017.

Rupali Mantri, S., R. Karyekarte Akshay, A. Ambore, Nitin and P. Kombbade Sarika. Prevalence of Methicillin Resistant Staphylococcus aureus in tertiary care Hospital, Central India. 2014. Vol. 3, Number 10, pp. 582-586.

Sachdev, D., Amladi, S., Nataraj, G.,Baveja, S., Kharkar, V., Maharajan, S., et al., (2003). An outbreak of Methicillinresistant Staphylococcus aureus (MRSA) infection in dermatology indoor patients. Indian J. Dermatol. Venereol. Leprol., 69:377 80.

Srinivasan A, Dick JD, Perl TM. Vancomycin resistance in Staphylococci. Clin Microbiol Rev 2002; 15: 430-8.

Weisblum B: Erythromycin resistance by ribosome modification. Antimicrob. Agents Chemother. 1985; 39: 577585.

Watanakunakorn C. Clindamycin therapy of Staphylococcus aureus endocarditis. Clinical relapse and development of resistance to Clindamycin, lincomycin and erythromycin. Am J Med 1976; 60: 419-25.

\section{How to cite this article:}

Krishnan, R., M. S. Harbade (Duthade), J. A. Iravane (Bajaj) and Gaikwad, A. A. 2019. MRSA and Inducible Clindamycin resistance in Staphylococcus aureus from Various Samples in A Tertiary Care Hospital. Int.J.Curr.Microbiol.App.Sci. 8(12): 14-19. doi: https://doi.org/10.20546/ijcmas.2019.812.003 